\title{
CAUSAS DE INTUBACIONES VIGILES REALIZADAS POR RESIDENTES DE ANESTESIA EN PACIENTES DEL HOSPITAL CARLOS VAN BUREN
}

\author{
Sofía Zúñiga San Martín ${ }^{1}$ Claudio Valencia Parada ${ }^{1}$, Bruno Caracci Pérez ${ }^{1}$, Carlos Narvaéz Moreno ${ }^{2}$ \\ Residente programa Anestesiología y Reanimación, Universidad de Valparaíso, Chile. \\ Docente programa Anestesiología y Reanimación, Universidad de Valparaíso, Chile. Jefe Servicio Anestesia.
}

Introducción: La intubación vigil es una competencia necesaria para el anestesiólogo actual y debe ser parte de un programa de residencia en la especialidad. Quisimos conocer las indicaciones más frecuentes de este procedimiento en el centro en donde se realiza la formación de vía aérea difícil de la universidad de Valparaíso.

Objetivo General: Determinar las causas más frecuentes de intubaciones vigiles realizadas por residentes de anestesia en pacientes que se someten a cirugía en el Hospital Carlos Van Buren. Material y Métodos: Se revisó la base de datos de pacientes de los residentes de la universidad de Valparaíso en donde se identificaron los pacientes que fueron sometidos a intubación vigil por becados entre el 01 de septiembre de 2013 y el 30 de agosto de 2016. Posteriormente se revisaron las fichas clínicas de los pacientes previamente identificados y se consignaron. Se registraron datos demográficos y motivo por el cual se indicó la intubación vigil. Todos los pacientes se sometieron al protocolo de nuestra institución que consiste en Atropina $1 \mathrm{mg}$ IM previo al procedimiento con posterior preparación de cavidades nasales y bucofaríngea con torulas de lidocaína $2 \%$ para luego iniciar TCI de remifentanil hasta llegar a un nivel de sedación Ramsey 2-3 con lo que se procedía a realizar la intubación. Para la intubación se utilizó videolaringoscopía, Fibrobroncoscopía o bonfils según decisión del operador.

Resultados: Se incluyeron 45 pacientes $(15 \%$ mujeres), con un promedio de edad de 51 años (IQR 37-58), IMC promedio 31,6. De todos los casos $3(6,6 \%)$ fueron de urgencias. En 33 pacientes $(73,3 \%)$ se utilizó fibrobroncoscopio, en $10(22,2 \% \%)$ Videolaringoscopio y en dos pacientes se utilizó bonfils $(4,5 \%)$. La causa más frecuente fue tumoral (20 pacientes, 44,5\%), luego fracturas maxilofaciales (10 pacientes, $22,2 \%$ ). En un 20\% (9 pacientes) se consideró que no existía indicación formal para intubación vigil y que se realizaron como entrenamieto para residentes. De las causas tumorales, el más frecuente es el tumor de lengua con 7 pacientes (35\% de las causas tumorales), seguido del tumor de laringe (25\%), y cáncer de Tiroides y metástasis cervical con un $10 \%$ cada uno.

Conclusiones: El conocimiento de las causas por las cuales se realizan intubaciones vigiles es importante para la toma de decisiones sobre como enfrentar una potencial vía aérea dificil en distintas situaciones. Sumado a esto es esencial conocer la epidemiología del centro donde se realizan los procedimientos y las dificultades que pueden tener las distintas patologías. Cabe destacar que al ser un centro docente existe una mayor exposición a los pacientes a intubación vigil, sin que necesariamente tengan una indicación formal para su realización, lo que presenta un dilema ético importante. En este sentido la supervición por parte de un docente entrenado cobra especial relevancia para mantener la seguridad del paciente en todo momento. 